\title{
EL TRATADO DE LIBRE COMERCIO (TLC): IMPLICANCIAS EN LAS EMPRESAS NACIONALES. «UN ENFOQUE MICROECONÓMICO»
}

\author{
Nicko. Gomero Gonzales \\ Docente de la Facultad de Ciencias Contables
}

\begin{abstract}
RESUMEN
En el presente artículo se hace uso de las herramientas macroeconómicas, como son: los costos, ingresos, precios domésticos, precios internacionales, aranceles, entre otros, para explicar claramente lo que sucedería con las empresas nacionales debido a la implementación del TLC. En el presente análisis se llega a determinar que si bien es cierto, el TLC podría impactar negativamente en la gestión de los negocios, ya que les restaria utilidades o beneficios extraordinarios, esto no implica que tomen la decisión de salir del mercado por este resultado. Muy por el contrario, existen estrategias macroeconómicas que permiten que estas organizaciones logren su reposicionamiento, pero en un mercado distinto estructuralmente, ya que la elevada competencia es la caracteristica más importante en la formación de este bloque económico.
\end{abstract}

Palabras clave: Precio internacional, precio doméstico o local, costo marginal, minimización de pérdidas y aranceles.

\section{INTRODUCCIÓN}

La globalización es un proceso que, según su tendencia, apunta a romper todo tipo de barreras que impidan el libre comercio entre los países del globo. ¿bueno?, ¿malo?, ¿modelo impuesto por las grandes corporaciones o países poderosos? Sobre este punto se podría emitir muchos juicios de valor. Pero de algo se debe estar seguro: La globalización apunta a consolidarse a nivel mundial. Evidencia de ello en el Perú, es la formacion del "AREA DE LIBRE COMERCIO PARA LAS AMÉRICAS» ALCA-y el Tratado Comercial que estápor entrar in vigencia con los Estados Unidos de Norteamérica.

Con el TLC se abre un espacio Importante para que las empresas nacionales zuedan colocar sus productos en el mercado más importante del mundo. La cantidad de consumidores potenciales en los Estados Unidos, con su poder de compra, garantizan que las empresas que se atrevan a exportar y a competir en este mercado tengan la opción de lograr importantes ganancias, que no solo sería ventajoso microeconómicamente, sino que sería la vía correcta para que el Perú pueda consolidar sus cuentas externas, nos estamos refiriendo a la Balanza Comercial, las RIN y el mercado de divisas.

Si bien muchos sectores no están conformes con la apertura de los mercados, dado que no se prepararon en su momento para competir externa e internamente, por esta posición no se debe paralizar un proceso que en el largo plazo; pues, más que costos, generará beneficios para sociedad. Las evidencias empíricas demuestran lo 
afirmado; según el Índice de Apertura Comercial publicado en el 2001 por el Fraser Institute de Canadá, los habitantes de los 10 países más abiertos al comercio ganan en promedio 7 veces más que las personas que viven en las 10 naciones más proteccionistas del mundo. Además, el libre comercio ha impactado en mayor medida a los países chicos que han abierto sus fronteras a los bienes y servicios de otras latitudes: entre los 10 países más libres se encuentran naciones pequeñas que no hace mucho eran pobres, como: Irlanda, Malasia y Singapur. Ésta es la prueba más contundente para avalar que el TLC es el instrumento para revertir estructuralmente la pobreza en el país.

En este contexto, es preciso señalar que el libre comercio no es una cuestión de simples números, de tasas de crecimiento o aumento en las exportaciones, sino del derecho fundamental de cada ser humano a intercambiar libremente el fruto de su labor con otros individuos a lo largo y ancho del planeta.

Si bien, la liberalización de comercio internacional es el camino correcto para salir del entrampamiento económico; entonces, por deducción, podemos afirmar, sin temor a equivocarnos, que los tratados comerciales son las estrategias correctas para reacomodarse en el nuevo escenario económico mundial, tal como es el TLC de Perú con Estados Unidos. Este bloque económico, generará impactos negativos, como cualquier medida de tipo económico, pero en definitiva sus bondades serán mayores para empujar las relaciones de comercio y así apuntar a mejorar sustancialmente el nivel de vida de la población en un largo plazo.

Las empresas que no se prepararon para afrontar el TLC, lamentablemente tienen que salir del mercado; pero no se podría decir que es un efecto negativo; todo lo contrario, es la oportunidad para que estas organizaciones que no puedan competir en el sector sujeto al TLC, busquen otras alternativas productivas donde sí sean competitivas, ésta es la forma cómo se optimizaría los recurso productivos, lo cual es ventajoso para todo tipo de economía.

En el presente artículo se desarrolla justamentelo que podría generar el TLC con los Estados Unidos a nivel microeconómico. La realización de este estudio permite, además, arribar a una serie de conclusiones y recomendaciones para que las empresas puedan competir con éxito este bloque económico, cuya característica principal es la elevada competencia.

\section{ENFOQUE MICROECONÓMICO DEL TLC}

Bajo un escenario de Tratado Comercial, donde se rompe todo tipo de barreras arancelarias al comercio internacional, las empresas afectadas tendrían que reacomodar sus procesos productivos, de comercialización y dentro de este último, los precios de sus productos que comercializan en el mercado doméstico; bajo este nuevo escenario, se dejaría de hablar de un precio doméstico, el cual tendría que ser sustituido por el término de precios globalizados, o internacionales $(\mathrm{Pi})$, es decir, los productos transables, serían cotizados tomando en cuenta las señales que proyecte el mercado mundial, cuyos principales componentes son la oferta y le demanda mundial (Om, Dm).

Como en el mercado nacional se tendría que transar los productos al nuevo precio internacional, muchas empresas verian mermadas sus márgenes de ganancia $o$, en el peor de los casos, originaría que tengan 
resultados negativos en su diferencial de ingresos y costos, con el consiguiente problema de que el primero de los resultados no le permita cubrir sus costos fijos como variables, y si este problema se convierte en estructural lo más lógico, a fin de no incurrir en pérdidas operativas, sería el cierre definitivo del negocio, de esta forma estaría dejando de maximizar pedidas.

Bajo el nuevo sistema de comercio, muchas empresas saldrían del mercado, pero hay que dejar en claro que la culpa no estaría por el lado del TLC, sino que este resultado se daría simplemente por que estas organizaciones no están capacitadas o preparadas para competir bajo un escenarıo sin barreras comerciales. Dado que los recursos productivos en todo tipo de economía son escasos, esta situación condiciona su óptima utilización; en este sentido, si las empresas con problemas operativos se quedan operando en el mercado, debido por ejemplo a un tipo de apalancamiento gubernamental, a sabiendas que son ineficientes, la sociedad como un todo perdería bienestar, ya que sería mejor que estas empresas salgan del mercado o, en todo caso, centren sus actividades en aquellas líneas de trabajo donde realmente sean eficientes.

Si bien es cietto que es preocupante que las empresas que operan con saldos en rojo en sus cuentas económicas y financieras, la preguntaría sería: ¿Hasta qué punto le convendría seguir operando, tomando en cuenta que el nuevo precio, bajo el efecto del TLC, no llegaría a cubrir sus costos operativos, específicamente los fijos como variables? Lo que comúnmente se escucha es que si una empresa tiene pérdida, la decisión correcta sería salir del mercado; tal como ya se mencionó, si este escenario ocurriera en términos estructurales o a largo plazo, la apreciación sería la correcta; pero si es en un corto plazo, la decisión tendría que estar por otro lado, es decir, lo más conveniente sería seguir produciendo, debido a que con esta forma se estaría minimizando pérdidas.

Por cierto, esta última decisión está sujeta a ciertas condiciones, la más importante es que los ingresos obtenidos por el negocio cubtan sus costos variables, o en todo caso, que el costo de contribución sea mayor a cero. Veamos un caso.

\subsection{Situación sin Tratado de Libre Comercio (TLC)}

Supongamos que la empresa ALFA produce pasta de ajos para ser comercializado en el mercado interno, siendo su estructura de costos como de ingreso tal como se describe en el gráfico $\mathrm{N}^{\circ} 1$.

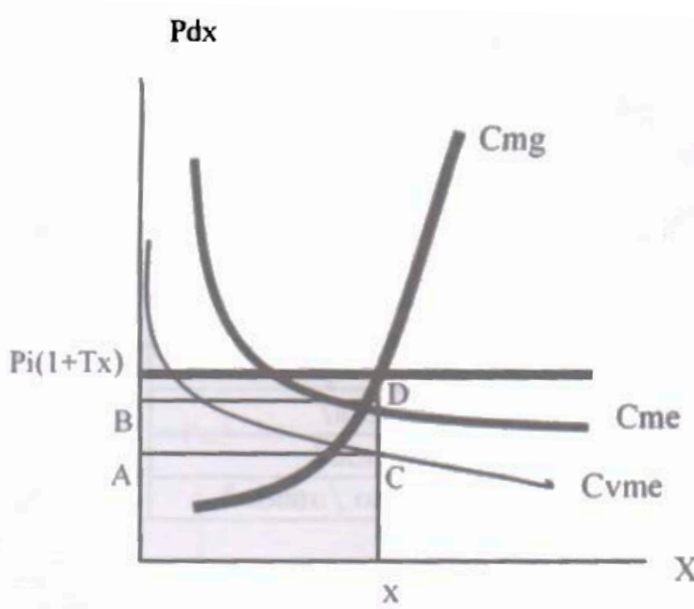

Gráfico N. ${ }^{\circ} 1$

Al analizar la situación de este negocio, se aprecia que el precio que maneja la empresa por comercializar su producto bajo un escenario sin TLC le permite cubrir el total de los costos productivos (costos variables más costos fijos) y obtener un 
beneficio extraordinario. El precio doméstico o nacional, en este caso, alcanza un límite condicionado por las barreras arancelarias $o$, en todo caso, por los mecanismos de protección del mercado nacional. El precio incluye: $P d=P i(1+T x)$; donde: $P d$ es precio domestico; $\mathrm{Pi}$, precio internacional; y $\mathrm{Tx}$, tarifas arancelarias. Cuanto más alta sea la tarifa o los aranceles, mayor será el margen de maniobra que tendrá el productor nacional para fijar el precio de su producto que comercializa en el mercado nacional, que si bien es cierto, este instrumento de protección le permite obtener un comportamiento positivo en sus cuentas de gestión, pero este beneficio termina por castigar a los consumidores, ya que estos últimos terminan pagando un mayor precio por el producto sujeto a protección.

¿Qué se entiende por beneficio extraordinario y cual será su compor- tamiento bajo un escenario con TLC? Se dice extraordinario porque al realizar el análisis de los beneficios, el concepto del costo explicito o contable es considerado como marginal, considerando para este análisis, por tanto, el del costo económico, que incluye el costo de oportunidad, es decir, lo que deja de ganar el empresario por estar en una determinada actividad comercial o productiva. ¿Cómo se puede diferenciar el costo contable con el económico? Para que quede claro este concepto se pone el siguiente cuadro a manera de ejemplo.

\section{Datos}

Producción: 100000 unidades de pasta de ajos Precio por unidad: US $\$ 2.2$.

Costo variables por unidad producida: US $\$ 1.5$ Costo fijo:US\$50 000

\begin{tabular}{|l|l|c|c|}
\hline \multirow{2}{*}{$\begin{array}{l}\text { Tipo de costos } \\
\text { /Ingreso }\end{array}$} & \multicolumn{2}{|c|}{ Contable } & Económico. \\
\cline { 2 - 4 } & $\begin{array}{l}\text { Producción: } \\
100000 \\
\text { unidades de } \\
\text { ajos }\end{array}$ & Total & Total \\
\hline Costo variable & 15 por unidad & 150000 & 150000 \\
\hline Costo Fijo & 50000 & 50000 & 500000 \\
\hline Costo de oportunidad & & & 20000 \\
\hline Costo totaI & & 200000 & 220000 \\
\hline ingreso total & & 220000 & 220000 \\
\hline Beneficio /utilidad & & 20000 & 0 \\
\hline
\end{tabular}

Tal como se muestra en el cuadro, en términos contables, las cifras de ingresos $\mathrm{y}$ egresos señala que habría una utilidad positiva; pero, en términos económicos, se iguala los costos con los ingresos, pero en este último caso hay que destacar que se cubre el costo de oportunidad del inversionista, obteniendo asi un beneficio normal equivalente en este monto, que es igual a US $\$ 20000$.

Ahora, si a estas cifras de ingresos y costos le adicionamos un $20 \%$ a este primer componente, el empresario, a parte de cubrir su costo de oportunidad, obtiene un 
resultado positivo adicional, originándose lo que se denomina un beneficio extraordinario; como es natural, todo empresario apunta a lograr este objetivo. En un mercado de elevada competencia, donde el precio es fijado por el libre mercado, obtener un resultado de este tipo se hace más complicado, ya que los márgenes tienden a reducirse, lo cual hace suponer que las empresas que se queden operando en el mercado bajo el TLC, que conceptualmente liberalizará el mercado, tendrían que sentirse satisfechos si logran obtener beneficios normales y no extraordinarios.

En caso que la empresa sea la dominante del mercado, esta situación sí podría ser aprovechada para apoderarse de la renta de los consumidores, cosa que se hace mas dificil cuando existe libre movilidad de factores, y un libre comercio como el que origina el TLC, que como se entiende generará justamente el rompimiento de los privilegios económicos de empresasque en un determinado momento gozaron de beneficios mono o cuasi monopólicos. Con el TLC, es dificil pensar que sobrevivan aquellas corporaciones económicas, que aprovechando el elevado grado de proteccionismo, mantenían mercados cautivos, con los consiguientes perjuicios de los consumidores.

Hay que entender que el TLC, solo limpia el mercado de impurezas, liquida aquellas empresas que nunca entendieron los nuevos paradigmas del manejo empresarial, para dar pase aquellas organizaciones con suficientes fortalezas para competir, no solo internamente, sino también en mercados de ultramar, y así convertirse en importantes generadoras de divisas, recurso financiero que sustentan las cuentas externas de un país, como son las Reservas Internacionales (RI) en poder de la autoridad monetaria ${ }^{1}$.

¿Cómo se puede graficar los beneficios normales y extraordinarios? Aquí la ilustración. Los ingresos y los costos se comportarían de la forma siguiente:

En el gráfico N. ${ }^{\circ} 2$ se presenta el caso de la obtención de beneficios normales, ya que en el punto $A$, donde se igualan los ingresos con los costos se cubre solo el Costo de Oportunidad; y en el gráfico $\mathrm{N}^{\circ} 3$ se da un benefició extraordinario representado por el área $\mathrm{ABCD}$. Es justamente con el TLC que esta área, por efectos de la competencia, comienza a diluirse, ya que el precio del

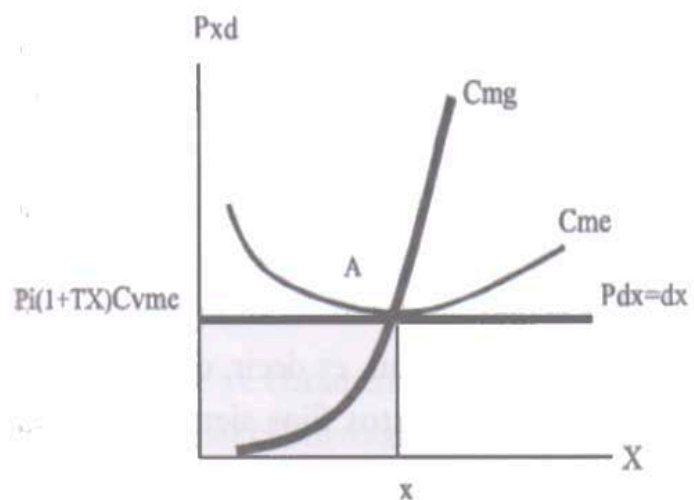

GráficoN. ${ }^{\circ} 2$

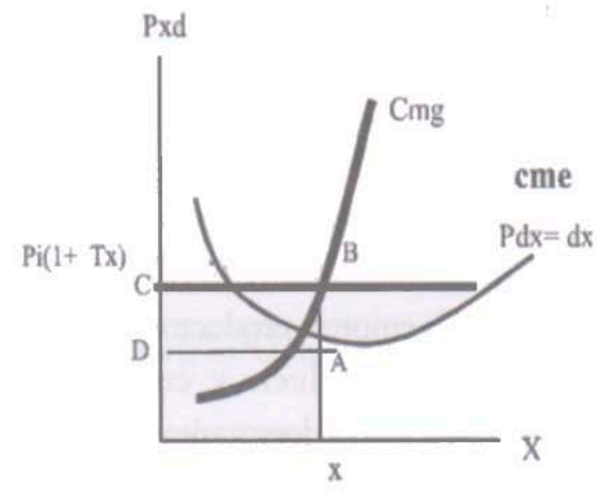

GráficoN. ${ }^{\circ} 3$

Banco Central de Reserva. 
producto tendría que ajustarse tanto a la oferta como a la demanda mundial.

\subsection{Situación con TLC}

Con TLC, las tarifas que se aplican a los productos importados se eliminan, por lo que los productores nacionales tendrán que ajustar sus precios de venta tomando en cuenta los precios de productos de importación. Por así decirlo, si el precio con tarifa era US $\$ 1.5$, a sabiendas que este impuesto es el $20 \%$, su eliminación implicará que este producto tenga que comercializarse internamente a US $\$ 1.2$ por unidad. Este es el precio máximo que se podrá cobrar por la pasta de ajos bajo un escenario de elevada competencia, como el que traerá consigo el TLC.

La relación funcional entre ambas variables es como se señala a continuación:

$$
P d x=F(P i)
$$

$P d x$ : Precio de los productos nacionales que tendrán que competir con los importados.

Pi: Precio del producto importado sin arancel.

$\mathrm{Si}$ antes de este acuerdo comercial las empresas domésticas manejaban un precio,

$$
P d x-P i(1+T x)
$$

Con el Tratado Comercial, va a hacerse $T x$ - arancel cero

$$
P d x=P i
$$

El precio nacional (Pdx) se hace igual al precio internacional (Pi). Más específicamente, si la empresa productora de pasta de ajos deseara producir y comercializar internamente su producto, rómo tendría que enfrentar a una oferta externa, que es igual a la cantidad importada, que se supone entrará al mercado en gran cantidad por el libre ingreso, el máximo precio que podrá cobrar por sus producto será igual a Pi (Precio internacional), tal como se señala en la formula, que es igual al precio nacional o doméstico.

Tal como se indica en el gráfico $N^{\circ} 4$, si antes del tratado comercial, debido a la protección, por el efecto precio, obtenía un beneficio extraordinario, al implementarse el acuerdo comercial, de un resultado positivo, podría pasar a un escenario negativo, con el consiguiente problema de no poder cubrir sus costos operativos. En este último caso se pueden presentar las siguientes situaciones:

a. Que el ingreso con Tratado Comercial le permita cubrir sus costos variables y parte de sus costos fijos.

\section{b. Que elingreso con tratado comercial no lepermita cubrir sus costos fijos como los variables.}

Ante estas dos situaciones, cuál debería ser la decisión correcta de la empresa que produce ajos en pasta.

En el primer.caso, en un corto plazo, a la empresa le convendría seguir operando. Veamos porqué. Si el ingreso que obtiene la empresa con el nuevo precio (con TLC), permite cumplir con los proveedores, con el pago de planillas (el componente variable), y otros tipos de costos y gastos considerados como variables, es más, si el ingreso estaría permitiendo cubrir parte de los costos fijos, la opción correcta sería seguir produciendo, aunque no se pueda lograr beneficios normales o extraordinarios. Hay que tener en cuenta, que si la empresa productora, dejara de producir, los costos fijos serían un desembolso inevitable que tendría que asumir, es decir, cierre o no la empresa, los costos fijos siempre estarán presentes en un corto plazo.

La empresa, bajo el nuevo escenario económico, al tomar la decisión de seguir operando estaría minimizando pérdidas, la 
cual es una forma de optimizar operaciones en un corto plazo.

Teniendo en cuenta este resultado para realizar el análisis del punto de equilibrio de la empresa, se llega a determinar que existiría un margen de contribución mayor a cero, que como bien se sabe, es el componente que permite cubrir los costos fijos.

El gráfico que explica este caso es el siguiente:

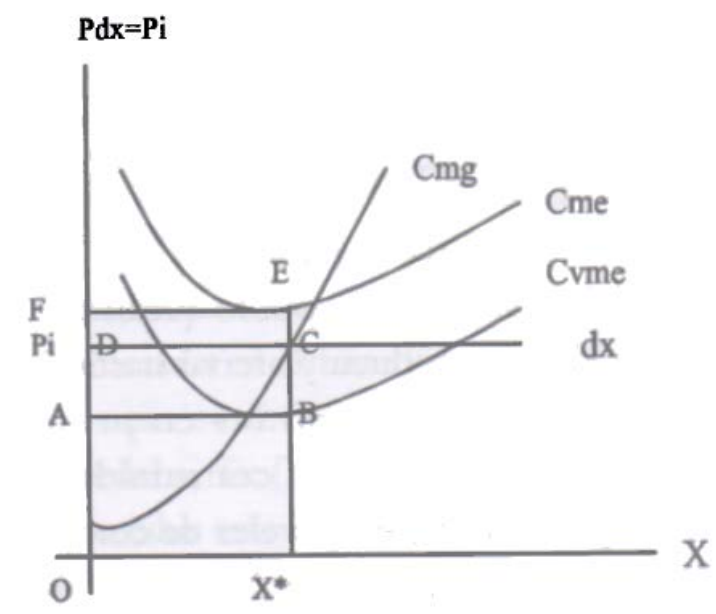

GráficoN. ${ }^{\circ} 4$

$\checkmark$ Costo Variable: $\mathrm{ABx} * \mathrm{O}$

Ingreso total con TLC $=\mathrm{DCx} * \mathrm{O}$

Costo fijo total : FEBA

Costo total : $\mathrm{FEx} * \mathrm{O}$

$\checkmark$ Proporción del costo fijo unitario que es cubierto por el precio: $\mathrm{EF} / \mathrm{CB}$

$\checkmark$ Los costos fijos que no son cubirto por el ingreso: FECD

$\checkmark$ Los ingresos $\left(\mathrm{DCx}^{*} \mathrm{O}\right)$ mayor que el costo variable $(\mathrm{ABx} * \mathrm{O})$

$\checkmark$ Beneficio Total: $\mathrm{FEx} * \mathrm{O}-\mathrm{DCx} * \mathrm{O}=$ menor a cero.

$\checkmark$ Margen de contribución:C-B

$\checkmark$ Punto de equilibrio en el punto de minimización de pérdida: FEBA/C-B

Como se ve claramente en el gráfico, el ingreso permite cubrir totalmente a los costos variables, pero no totalmente a los costos fijos, por lo que la mejor alternativa es que siga operando la planta de pasta de ajos, por el principio de minimización de pérdidas.
En el segundo caso, definitivamente la única opción que le quedaría al negocio sería salir del mercado, ya que los ingresos que obtendría, debido a la aplicación del Tratado Comercial, no le permitiría cubrir los componentes de costos que están ligados directamente al proceso de producción, a la misma vez no habrían los recursos suficientes para cubrir los costos fijos. En este caso minimizaría pérdida, cerrando el negocio.

Hay que señalar que en ambos casos, quien ha invertido en este negocio, no logra cubrir su costo de oportunidad, por lo que sería apropiado decir que sus beneficios económicos son negativos.

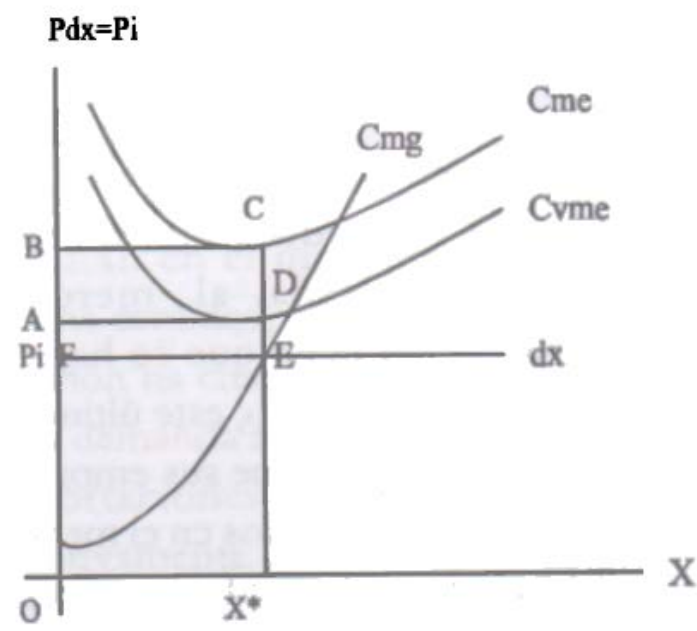

\section{Gráfico N: 5}

$\checkmark$ Costo Variable: ADx*O

$\checkmark$ Ingreso total con TLC $=\mathrm{FEx} * \mathrm{O}$

$\checkmark$ Costo fijo total:BCDA

$\checkmark$ Costo total: $\mathrm{BCx} \mathrm{x}^{*} \mathrm{O}$

$\checkmark$ Proporción del costo fijo unitario que es cubierto por el precio: (como mayor que el Precio) margen de contribución es menor que cero.

$\checkmark$ Los costos fijosque no son cubiertos por el ingreso: BCDA

$\checkmark$ Los ingresos $\left(\mathrm{FEx}^{*} \mathrm{O}\right)$ menor que el costo variable $(\mathrm{ADx} * \mathrm{O})$

$\checkmark$ Beneficio Total: $\mathrm{FEx} * \mathrm{O}-\mathrm{BCx} * \mathrm{O}=$ menor a cero.

$\checkmark$ Margen de contribución: $D-A=C v m e$ menor al precio

Si el negocio presentara la estructura de costos como ingresos señalados en el gráfico 
$\mathrm{N} .^{\circ} 5$, lo único que le queda para minimizar pérdidas es salir del mercado, es decir, su ineficiencia lo obliga a cerrar definitivamente.

Este caso se puede presentar para muchos negocios, que en un primer momento no se prepararon para competir en mercado aperturados, como ya se dijo en un principio; la fuerzas del mercado lo desplazan del sector donde está operando, y lo obligan a buscar otras alternativas productivas que impliquen utilizar con mayor eficiencia y eficacia los recursos productivos. Como se dice: el mercado es el mejor asignador de recursos, y en este caso estaría cumpliendo esa función, el cual es beneficioso para la sociedad como un todo.

Por otro lado, como con el TLC se liberalizará el comercio internacional, los productos que se producen localmente tendrán libre entrada al mercado internacional, o del país que se haga el tratado comercial; asimismo, este último le dará la oportunidad para que sus empresas puedan colocar sus productos en el merado nacional libre de toda tarifa comercial, situación que dinamizará el comercio bilateral, con el consiguiente impacto en las exportaciones e importaciones de ambas economías.

En la primera parte de este trabajo se ha hecho el análisis del impacto que tendría el TLC en las empresas cuya estructura productiva no está preparada para enfrentar este nuevo escenario comercial. ¿Pero que pasará con los consumidores y el Gobierno? ¿Saldrían ganando o estarían peor que antes? Para responder a estas preguntas se utilizará la demanda y oferta nacional como principales herramientas microeconòmicas de análisis, complementado con los precios internacionales y domésticos. Supongamos que el sector afectado sea el algodonero. ¿Qué sucederá con los productores de este producto con la implementación del TLC?

Hagamos el siguiente análisis:

\section{Variables utilizadas}

Xsn: Producción nacional

$X d n$ : Demanda nacional

Pi $(1+T x):$ Precio $\sin T L C$

Pi: Precio con TLC: que es igual al precio internacional que está libre de tarifas

Antes de la aplicación del acuerdo comercial, los productores de algodón, que gozaban de cierta protección con las tarifas, contaban con un techo límite de $\mathrm{Pi}(1+\mathrm{Tx})$, para manejar sus precios, por lo que estarán en capacidad de producir y ofertar hasta Xo de este producto, como las empresas textileras representan sus consumidores reales y potenciales, sus niveles de compra o demanda llegan hasta X1, como la demanda excede a la producción de algodón, la brecha, que ésta representada por el segmento $A B$, será cubierto vía importaciones, igual a (X1-Xo). Como se aprecia, los productores de algodón deben de sentirse satisfechos con la protección, pero no desarrollan suficientes fortalezas para abastecer al mercado textilero, cuya demanda como ya se mencionó tiene que ser cubierta con oferta externa.

Al aplicarse el TLC, que implica eliminar las tarifas comerciales, el techo límite que tienen los productores nacionales del algodón será igual al precio internacional (Pi). A este nuevo nivel de precio, la producción de algodón pasará de $\mathrm{X} 0$ a X3 . La pregunta es ¿Por qué baja la producción nacional de algodón? La respuesta es simple. Por que existen productores que no tienen capacidad de operar bajo este nuevo 
escenario económico, es decir, los precios internacionales que son equivalentes al precio doméstico no le permiten cubrir sus costos variables, por lo que el único camino que tienen para no maximizar pérdidas es salir del mercado, más específicamente saldrán del mercado por ineficientes. Lo que le quedaría es que sus recursos productivos sean orientados a la producción de productos alternativos, donde puedan tener ventajas comparativas o competitivas, en este último caso estarían dando óptimo uso a los recursos productivos. De ineficientes en el sector algodonero pasarian a ser eficientes, por ejemplo en la siembra de maiz amarillo.

Otros de los agentes económicơs que se ve afectado es el Estado, si antes del TLC, tal como se muestra en el gráfico $\mathrm{N} .^{\circ} 6$, recaudaba, Tx (X1-Xo), con el TLC, dejará de percibir este ingreso tributario, ya que la tarifa por efectos del acuerdo es igual a cero. El área que deja de percibir el Estado es igual a $\mathrm{ABCD}$. Como contrapartida a este resultado, los consumidores saldrian ganando con el acuerdo; es decir, los textileros aumentarían su consumo de algodón de X1 a X2, motivado por un menor precio en el mercado algodonero.

Como el TLC ha originado que la oferta de algodón disminuya de Xo a X1 y la demanda ha aumentado de X1 a X2, aumenta el flujo de importaciones. Ahora, si las exportaciones no crecen en mayor magnitud que las importaciones, el resultado inmediato será la obtención de un saldo negativo en la balanza comercial de este sector, con la consiguiente salida de divisas y debilitamiento de las RIN.

¿Que pasaría si el sector textilero se reactiva debido a la demanda de telas de los

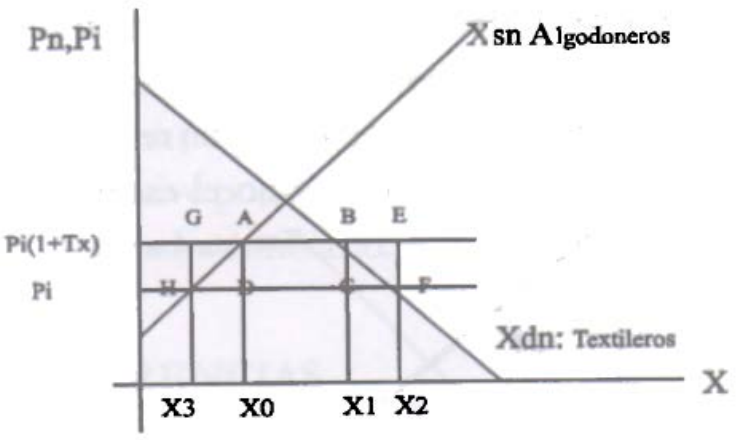

Gráfico N. ${ }^{\circ} 6$

confeccionistas? ¿Cual sería el impacto en el mercado algodonero? ¿Tendría un impacto positivo en los productores locales? Definitivamente, si los productores locales de algodón, no logran recomponer su estructura de costos, lo que pase en el sector de confecciones y textileros no le generará ningún impacto favorable. Más específicamente, los mismos productores seguirán en el mercado produciendo la misma cantidad, pero como la demanda de algodón ha crecido, este mayor dinamismo de la demanda será sustentado con mayores importaciones de algodón, impactando negativamente en las cuentas externas del país. El gráfico N. ${ }^{\circ} 7$ ilustra la idea presentada. La nueva cantidad importada de algodón por los textileros será X4-X3. Esta situación ilustra como la reactivación productiva de un sector ocasionará un problema de divisa en la economía, es decir, se crece pero quemando divisas, por el hecho que las empresas locales no cuentan con capacidad de oferta. Si el TLC conlleva a que las importaciones crezcan más rápidamente que las exportaciones, como ya se dijo, el impacto negativo en las RIN es inmediato, su control depende del BCR.

Por último, como el precio doméstico está dado en el mercado, lo cual hace que la empresa se convierta en tomadora de precio, 
las únicas variables que tiene opción de cambiar son los costos productivos, por lo que sus estrategias para pasar de un resultado negativo a otro positivo, bajo el escenario

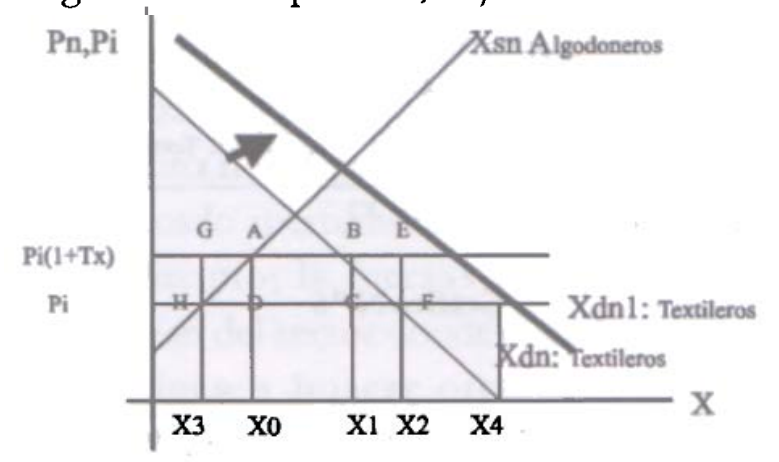

Gráfico $\mathbf{N} \cdot{ }^{\circ} 7$

del TLC, es recomponer los elementos que lo constituyen. Algunas medidas podrían ser las siguientes:

- Recomponer su política de compra de insumos y materias primas

- Ajustar la cantidad de personal de la empresa. Revisar su manual de funciones y rediseñar su estructura organizacional.

- Realizar Alianza Estratégica con los proveedores

- Revisar sus flujograma de procesos: administrativos como productivos. Eliminar aquellos cuya relevancia es mínima en la empresa, con lo cual se liberaría recursos productivos.

Al tomar estas medidas, dado el TLC, con un precio internacional vigente en el mercado nacional (Pi), la empresa pasaría de una situación como se presenta en la figura $\mathrm{N}^{\circ} 8$ a otra como se presenta en el gráfico $\mathrm{N}^{\circ} 9$, con el consiguiente desplazamiento hacia abajo de los costos de producción (Cmeo A Cmel). Se consigue un resultado favorable por la implementación de medidas que atacan a los costos de producción, los precios no tienen ninguna injerencia para lograr este resultado, ya que su nivel depende no del negocio, sino del mercado, situación que lo convierte en una variable exógena para la empresa.

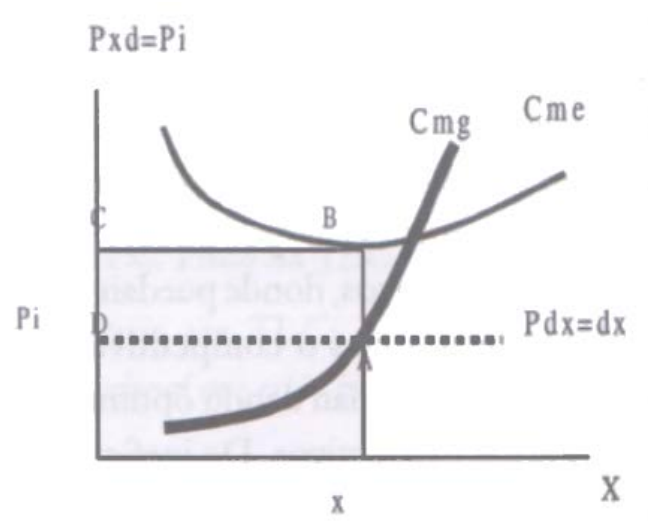

Gráfico N. ${ }^{\circ} 8$

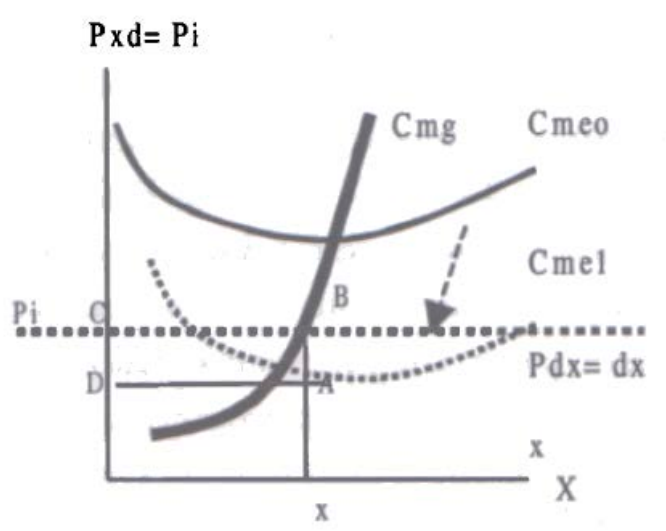

Gráfico $\mathbf{N}^{\circ} 9$

\section{CONCLUSIONES}

1. La implementación de mecanismos de control o de protección a las empresas nacionales genera que éstas obtengan beneficios que muchas veces son irreales, lo cual, a la larga, los convierte en ineficientes.

2. El TLC originará que muchas empresas salgan del mercado, motivado más que todo por sus deficiencias como organización, cuya debilidad no le permitirá operar en mercados aperturados o liberalizados. 
3. Si las empresas, como producto del TLC, llegan a obtener resultados negativos en sus utilidades, les convendría seguir operando en el mercado, siempre y cuando estén en capacidad de cubrir sus costos variables, caso contrario la mejor opción es salir del sector donde están operando.

4. Si el TLC, origina que empresas débiles salgan del mercado, la sociedad ganaría como un todo, ya que se estaría optimizando la utilización de los recursos productivos. Estas empresas desplazadas podrían buscar actividades cuyas fortalezas les permitan competur en el mercado.

5. Bajo el TLC, se dinamizará las importaciones de productos; si esta no es compensada con un mayor flujo de exportaciones, podría comprometer las cuentas externas, como son las RIN, inclusive podría afectar la estabilidad del mercado cambiario.

\section{REFERENCIAS}

KAFKA, Folke. Universidad del Pacífico. CIUP. Lima-Perú.

VARIAN, H. (1999) Microeconomía Intermedia. 5. ${ }^{\mathrm{a}}$ ed., Barcelona, Antoni Bosch (editor), 1999.

NICHOLSON, W. Teoria Microeconómica. 6. ed., Madrid, Edit. McGraw-Hill, 1997. STIGLITZ, J. Microeconomia. Madrid, Edit. Ariel, 2004. 